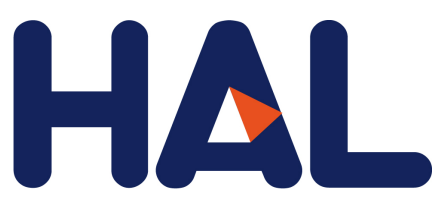

archives-ouvertes

\title{
On the threshold crack opening effect on the intrinsic permeability of localized macro-cracks in concrete samples under Brazilian test conditions
}

Giuseppe Rastiello, Stefano Dal Pont, Jean-Louis Tailhan, Pierre Rossi

\section{- To cite this version:}

Giuseppe Rastiello, Stefano Dal Pont, Jean-Louis Tailhan, Pierre Rossi. On the threshold crack opening effect on the intrinsic permeability of localized macro-cracks in concrete samples under Brazilian test conditions. Mechanics Research Communications, Elsevier, 2018, 90, pp.52-58. 10.1016/j.mechrescom.2018.04.008 . hal-02085758

\section{HAL Id: hal-02085758 \\ https: / hal.archives-ouvertes.fr/hal-02085758}

Submitted on 23 Oct 2019

HAL is a multi-disciplinary open access archive for the deposit and dissemination of scientific research documents, whether they are published or not. The documents may come from teaching and research institutions in France or abroad, or from public or private research centers.
L'archive ouverte pluridisciplinaire HAL, est destinée au dépôt et à la diffusion de documents scientifiques de niveau recherche, publiés ou non, émanant des établissements d'enseignement et de recherche français ou étrangers, des laboratoires publics ou privés. 


\title{
On the threshold crack opening effect on the intrinsic permeability of localized macro-cracks in concrete samples under Brazilian tests conditions
}

\author{
Giuseppe Rastiello, ${ }^{\mathrm{a}, *}$, Stefano Dal Pont ${ }^{\mathrm{b}}$, Jean-Louis Tailhan ${ }^{\mathrm{c}}$, Pierre Rossi $^{\mathrm{c}}$ \\ ${ }^{a}$ DEN - Service d'études mécaniques et thermiques (SEMT), CEA, Université Paris-Saclay, 91191 Gif-sur-Yvette, France \\ ${ }^{b}$ Grenoble-INP, UJF, CNRS UMR5521, Laboratoire 3SR, Domaine Universitaire, BP53 38041 Grenoble, Cedex 9, France \\ ${ }^{c}$ IFSTTAR, Université Paris-Est, Facultée de Médecine secteur-Nord, Bd. P. Dramard, 13916 Marseille, France
}

\begin{abstract}
The Brazilian (indirect tensile strength) test is widely adopted for studying the permeability evolution of single localized macro-cracks in concrete samples. In most of these studies, the existence of a threshold crack opening effect on the crack permeability is argued, in particular when permeability measurements are performed on previously cracked samples (i.e., on residual cracks). Based on recent experimental results obtained by Authors, the present contribution aims to show that performing permeability measurements in real-time with the crack opening process (i.e., under loading) ensures improving the results quality and makes their interpretation less ambiguous. It is shown that no threshold crack opening effect is detected in the explored range of crack opening displacement. Although presented results do not allow to conclude concerning the existence of such an effect, they point out several critical aspects concerning the interpretations usually provided. In particular, based on the comparison between present and reference results, it is proposed to interpret literature observations as a direct consequence of some artifacts inherent to standard experimental procedures, such as the impossibility of distinguishing between crack and porous medium contributions to the measured mass flow rate, crack re-closure due to unloading and/or self-healing phenomena, an inaccurate estimation of the crack opening displacement corresponding to flow measurements.
\end{abstract}

Keywords: Crack permeability, Localized crack, Concrete, Brazilian test, Threshold crack opening effect

\section{Introduction}

Several experimental studies aiming to analyze the effect of localized macro-cracks on the transfer properties evolution of concrete were presented in the literature [11]. In the majority of these works $[28,2,9,19,1,8]$ a mechanical Brazilian (indirect tensile) test is used to obtain a single crack centered on the sample, while transfer properties are characterized after unloading the cracked specimen (i.e., transfer properties measurements are performed on residual cracks).

Although these approaches have the advantage of allowing the use of standard permeability measurement techniques, some parasite effects cannot be avoided. Indeed, cracks tend to re-close after the specimen unloading and during the transfer test due to multi-physics effects (e.g. elastic recovery, self-healing phenomena, etc.). As a consequence, no direct correlation between the measured flow and the crack opening displacement can be obtained.

As first proposed by Boulay et al. [5] for electrical resistance measurements, most of these parasite effects can be

\footnotetext{
* Corresponding author.

Email address: giuseppe.rastiello@cea.fr (Giuseppe Rastiello)
}

strongly reduced by monitoring transfer properties evolution under loading (i.e., in real-time together with the crack opening process). Drawing from this idea, Rastiello et al. [21] developed an experimental protocol for studying the water flow evolution through a concrete specimen under Brazilian loading.

Based on the results of a wide experimental campaign, a modified "parallel-plates model" was proposed. According to this schematization, the crack was approximated as the space comprised between two parallel and smooth plates $[25,29,30]$. Their distance (i.e., the crack opening) was estimated as the mean value of the mean crack openings computed over the two plane faces of the sample ${ }^{1}$. This allowed to directly relate the so-called crack permeability $k_{c}$ to the mean crack aperture $\bar{a}$ (Fig. 1 ), through the introduction of a non-linear opening-dependent phenomenological corrective factor accounting for the main causes of deviation (roughness of the crack walls, tortu-

\footnotetext{
${ }^{1}$ This quantity was shown to properly represent crack transmissivity for the whole specimens (diameters ranging from 110 to 250 $\mathrm{mm}$ and thickness ranging between 50 and $130 \mathrm{~mm}$ ) tested in the framework of the experimental campaign. Furthermore, the sample thickness did not significantly influence the crack permeability aperture relationship. For traversing cracks, this was interpreted as the evidence of the absence of scale effects with respect to the crack length.
} 
osity of flow-paths, contact areas, ...) between flow in real cracks and the Poiseuille's flow assumption. This was possible because, due to the rapidity of the test, the crack flow only was monitored and no self-healing phenomena occurred.

These experimental results put in evidence that the crack flow intensity tended to increase monotonically in the whole range of explored crack apertures: mean crack apertures $\bar{a} \in[15 \div 160] \mu \mathrm{m}$, and crack apertures at mid-height of the faces of the sample $a_{m} \in[30 \div 300] \mu \mathrm{m}$. This is however in contrast, at least apparently, with a recurrent argument in the technical literature concerning cracked concrete (see e.g. [28, 2, 19]): the existence of a so-called "threshold crack opening effect" for the transfer properties of the crack. Classically assumed between 25 and $100 \mu \mathrm{m}$, this quantity can be seen as the limit crack aperture below which the fluid flow is not influenced by the presence of the crack. Such an aspect is of paramount importance when estimating the safety level (e.g. with respects to leaks) of concrete structures.

Opening a discussion about the experimental determination and meaning itself of such a quantity is the aim of the present paper. Based on comparisons among results by [21] and literature results concerning water flows in cracking/cracked concrete samples, we show that its existence cannot be adequately investigated using standard experimental methods. The above mentioned authors provided the best possible results with the experimental methods/tools in their possession. However, we point out that using more advanced/recent experimental techniques allows improving the results quality and makes their interpretation less ambiguous. In other words, we do not rigorously demonstrate that such threshold effect does not exist, but point out several critical technical/theoretical aspects that may affect interpretations provided in reference research works. The comparison among air flow results is finally the occasion for stressing a further possible parasite effect that often occurs in Brazilian tests.

\section{Experimental method}

The experimental protocol for studying the real-time evolution of water flow through a cracking sample under diametral loading is presented briefly. For a complete and detailed description of the experimental setup and testing procedure one can refer to [21].

\subsection{A three-steps protocol}

A Brazilian test (axial splitting test) is used to obtain a single crack, centered on the faces of the cylindrical sample. It is $d_{s}$ in diameter and $t_{s}$ in thickness.
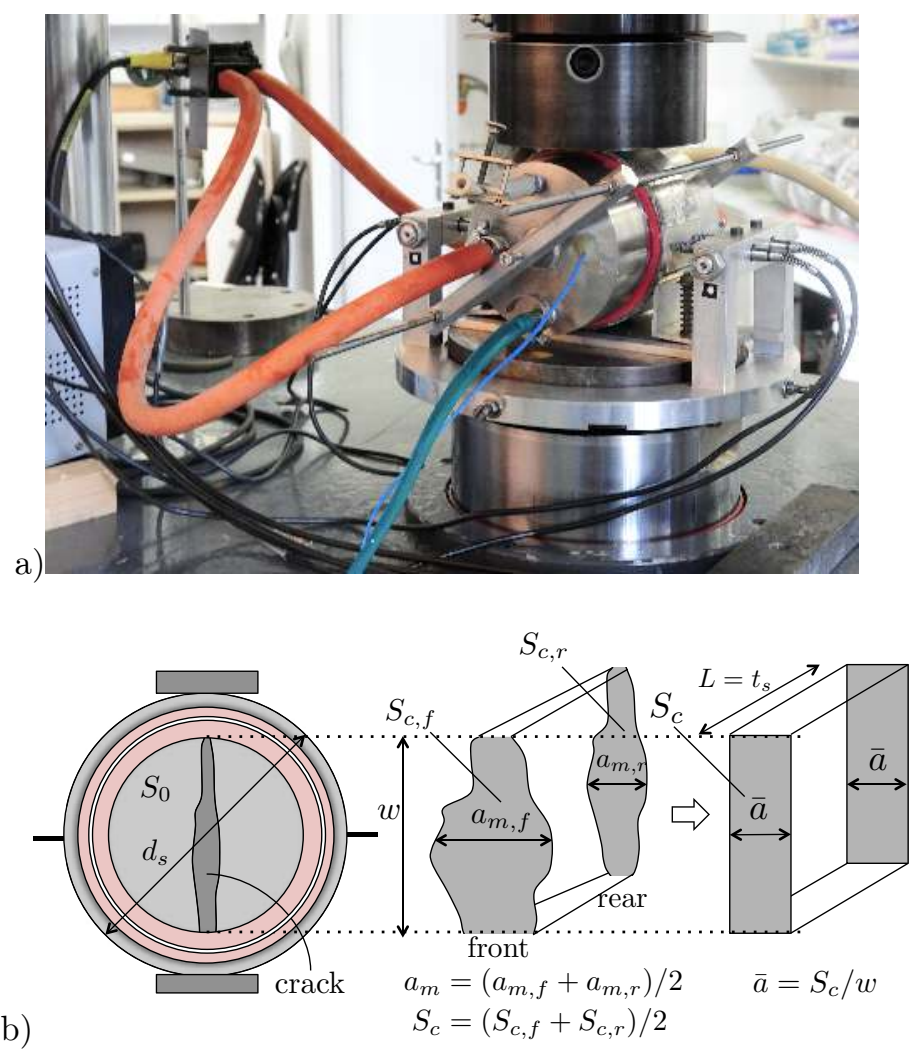

Figure 1: a) Experimental equipment for permeability measurements under loading (HM protocol), b) Effective crack geometrical properties: surface of the sample exposed to the fluid pressure $\left(S_{0}\right)$, mean value of the crack openings at mid-height of the faces of the sample $\left(a_{m}\right)$, effective crack surface $\left(S_{c}\right)$ and mean crack opening $(\bar{a})$.

In order to avoid mechanical instabilities (snap-back, snapthrough) occurring in the force post-peak phase, the diametrical load is controlled indirectly through the mean diameter variation $\left(\Delta d_{s}\right)$ of the sample. This quantity is computed in real-time during the test as the mean vaue of the diameter variations measured by two couples of Linear Variable Displacement Transducers (LVDTs) symmetrically placed $( \pm 15 \mathrm{~mm})$ with respect to the mean vertical cross section of the sample.

The three-steps protocol can be summarized as follows:

(i) Hydro-mechanical (HM) tests. Two water filled vessels were placed on the two plane faces of the saturated sample. During the test, the load was controlled to ensure a constant rate of change in $\Delta d_{s}$. The diameter variation was then blocked at regular intervals. For each $\Delta d_{s}$ level, different levels of the pressure difference $(\Delta P)$ were imposed between the two plane faces of the sample for a long enough duration to attain steady-state flow conditions. The mass flow rate through the crack $\left(Q_{c}\right)$ was then monitored in real-time and put into relation with global and crack opening displacements.

(ii) Mechanical (M) tests. During HM tests the sole crack geometrical information available from mea- 
sured displacements were the crack apertures at midheight of the faces of the sample. They were computed from force - displacement curves as proposed in $[5,21]$. To complete this information, the crack geometry evolution under loading was studied through a further series of purely mechanical Brazilian tests where a Digital Imaging Correlation (DIC) technique was used to follow the displacement field and crack opening evolutions on the plane faces of the sample.

(iii) Coupling HM and $\mathbf{M}$ results. Flow rate measurements and crack geometrical properties were coupled by using $\Delta d_{s}$ as an exchange variable. In particular, assuming an incompressible laminar flow, the transmissivity of the crack was computed $\mathrm{as}^{2}$ :

$$
T_{c}=-\frac{\mu}{\rho} \frac{L}{\Delta P} Q_{c}
$$

and the crack permeability estimated as:

$$
k_{c}=T_{c} / S_{c} \quad S_{c}=S_{c}\left(\Delta d_{s}\right)
$$

where $\mu$ was the dynamic viscosity of water, $\rho$ its density, $\Delta P=P_{2}-P_{1}$ was the pressure change between the two faces of the sample, $P_{1(2)}$ was the corresponding inlet (outlet) pressures. The length of the crack $(L)$ was supposed equal to the thickness of the sample and the mean cross section of the crack $\left(S_{c}\right)$ was computed for each $\Delta d_{s}$ level via DIC results. In that case, it is assumed that the presence of the permeability measurement system does not influence the cracking process.

\subsection{Typical results}

Typical crack transmissivity evolutions with respect to the mean diameter variation of the sample, the mean crack opening displacement at mid-height of the sample $\left(a_{m}\right)$ and the mean value of the mean crack opening $(\bar{a})$ over the faces of ordinary concrete (OC) samples ${ }^{3}$ (110 $\mathrm{mm}$ in diameter and 50, 90 and $130 \mathrm{~mm}$ in thickness) are provided in Fig. 2.

These results show clearly that $T_{c}$ tends to increase in the whole range of explored crack apertures and no threshold effects are present.

It should be noticed that the mechanical procedure commonly adopted for cracking generation limits the experimental results that are available for small crack openings.

\footnotetext{
${ }^{2}$ Imposed $\Delta P$ levels were small enough to consider negligible any flow nonlinearities associated with recirculation phenomena induced by solid-fluid interactions inside cracks [7, 30].

${ }^{3}$ Using a Digital Imaging Correlation technique, [21] showed that $\bar{a} \approx a_{m} / 2$ for any loading level. A very similar correlation can be derived from optical measurements performed in [19] on unloaded samples.
}

Indeed, when using the Brazilian test, a macro-crack propagates in the sample after reaching the load peak. For the $\mathrm{OC}$ formulation that is considered in this work, this condition is reached when $\Delta d_{s} \approx 20-25 \mu \mathrm{m}$. For that reason, transfer measurements were performed only for $\Delta d_{s} \geq 50$ $\mu \mathrm{m}$.

In $[5,21]$ it was evidenced that once the load peak is attained, the sample is split into two elastic blocks and a macro-crack traverses the sample. This was observed by using a Digital Imaging Correlation technique in purely mechanical Brazilian tests, and is indirectly confirmed by flow measurements in hydro-mechanical experiments.

Remark 2. These results are presented in slightly different form [21]. Using $T_{c}$ as transfer properties variable allows presenting the experimental results based on direct measurements only, without any assumptions concerning the crack geometry. Furthermore, in this way, experimental results can be easily used for validating mesoscopic and macroscopic numerical modeling strategies of fluid leakage in cracking concrete [23, 22, 10, 15, 20, 3].

\section{Threshold crack opening discussion}

The threshold crack opening discussion is started considering water leakage. Experimental results obtained in [21] are compared to results published in reference works concerning water flow in cracked concrete. For the sake of simplicity, we refer to results by Wang et al. [28], but analogous qualitative/quantitative considerations hold for other similar works $[2,19]$. By this way, different considerations can be introduced concerning: (i) the variables to be used to interpret experimental results, (ii) the representativity of crack opening displacements measurements in standard experiments and (iii) the meaning itself of such a threshold crack opening effect.

\subsection{What transfer properties variable?}

The first consideration concerns the choice of a variable for properly representing transfer properties of cracked samples.

In the whole cited works, the "apparent permeability coefficient" $\left(k_{\text {app }}\right)$ of the cracked sample was used. The term "apparent" is used to denote that once the sample is cracked no homogenized quantities can be defined. Due to the adopted experimental techniques, in these works, it was however impossible to distinguish among crack and porous medium flow contributions. For that reason a single $k_{\text {app }}$ value was computed as a function of the total mass 

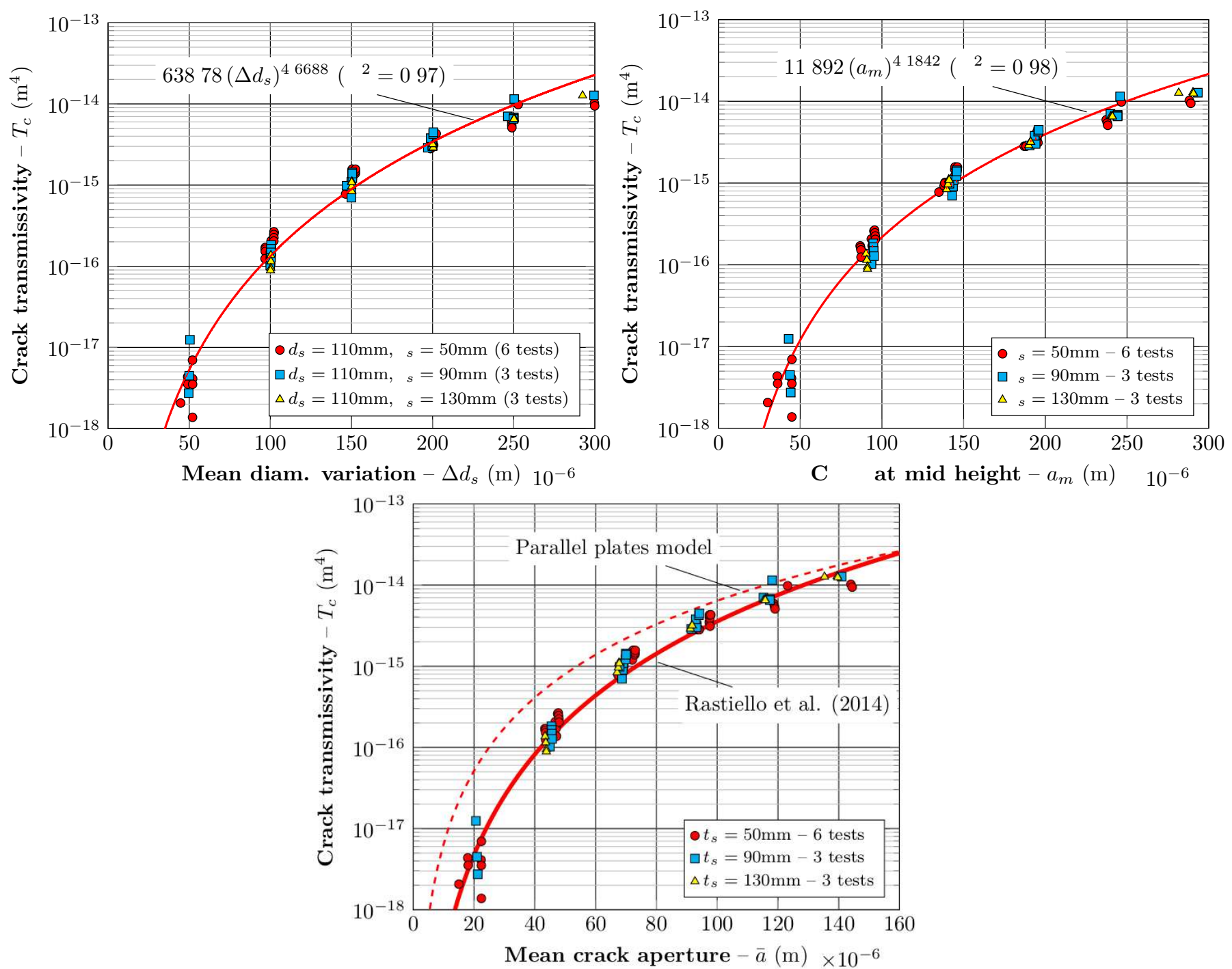

Figure 2: Transmissivity $T_{c}$ evolution with respect to (a) the mean diameter variation of the sample $\Delta d_{s}$, (b) the mean value of crack openings at mid-height of the faces of the sample $a_{m}$, (c) mean value of the mean crack openings on the faces of the sample. Samples are 110 mm in diameter and 50, 90 or $130 \mathrm{~mm}$ in thickness.

flow rate $(Q)$. By assuming the Darcy's law for laminar flow as valid one had:

$$
Q=-k_{\mathrm{app}} S_{0} \frac{\rho}{\mu} \frac{\Delta P}{L}
$$

where $S_{0}$ was the surface of the portion of the plane face of the sample which was exposed to the fluid pressure.

Now, the cracked specimen can be geometrically schematized as an homogeneous medium crossed by a rectilinear crack oriented along the direction of the macroscopic pressure gradient. For a quasi-unidirectional laminar flow both in the porous medium and the crack, and considering as negligible any fluid exchanges between these systems ${ }^{4}$, the

\footnotetext{
${ }^{4}$ This assumption seems reasonable in light of the permeability difference between the two systems.
}

total mass flow rate can be also written as:

$$
Q=Q_{0}+Q_{c}=-\left[k_{0} S_{0}+k_{c}(a) S_{c}\right] \frac{\rho}{\mu} \frac{\Delta P}{L}
$$

where $Q_{0}$ is the porous medium mass flow rate, $Q_{c}$ is the crack contribution and $k_{0}$ is the intrinsic permeability of the sound material. By comparing Eqs. (3) and (4) it is straightforward verifying that:

$$
k_{\text {app }}=k_{0}+\frac{S_{c}}{S_{0}} k_{c}(a)
$$

To consider the effect of the crack on the evolution of $k_{\text {app }}$ throughout the cracking opening process (for this simplified model), it is useful to introduce the ratio of the apparent permeability to its initial value (i.e., the intrinsic permeability of the sound medium):

$$
\phi^{w}=\frac{k_{\mathrm{app}}}{k_{0}}=1+\frac{S_{c}}{S_{0}} \frac{k_{c}(a)}{k_{0}} \geq 1
$$


According to this definition, the threshold crack opening can be seen as the larger $a$ value such that $\phi^{w}$ tends to unity (or, alternatively, as the opening level corresponding to a change in convexity of the $\phi^{w}(a)$ relationship).

Based on Eq. (6), it is however immediate to put into evidence the physical inconsistency of any discussion about transfer properties of cracks in terms of "apparent / homogenized" quantities of the cracked sample. Indeed, for a given crack aperture $(a>0)$ and extent (i.e., $k_{c}(a)>0$ and $\left.S_{c}>0\right)$, the higher are $k_{0}$ and/or $S_{0}$ the lower is the attained $\phi^{w}$ value. In other words, if the porous medium contribution $Q_{0}$ is large enough, the ratio $Q_{c} / Q_{0} \rightarrow 0^{+}$ even if $Q_{c}>0$ (Fig. 3). As a consequence, one could argue of some apparent threshold opening level, even through the crack flow is not negligible.

Remark 3. The apparent permeability coefficient nothing says about some intrinsic crack properties. Instead, it is crucial to distinguish between the porous medium and crack transfer properties properly. For instance, by trying to experimentally discriminate between the contributions to the total mass flow rate deriving from the crack and surrounding medium flows. In this way, one could estimate two distinct intrinsic permeability coefficients $\left(k_{0}\right.$, $\left.k_{c}\right)$, as it is classically done in the Rock Mechanics context $[29,30,4]$. This was done, for instance, by [1] and [8]. Both authors were able to measure the $Q_{c}$ contribution only by means of rapid permeability tests. Notice however that permeability measurements were performed on unloaded samples (residual cracks), which may partly explain the high dispersion of their experimental results.

Remark 4. Representing the transfer properties of the cracked sample through homogeneized quantities can also lead to other ambiguities. For instance, re-analyzing the experimental data presented by Picandet et al. [19] for air flows through OC samples under brazilian loading, Jourdain [12] argued that the results presented for large crack opening levels $\left(a_{m} \approx 100 \mu \mathrm{m}\right)$ could be affected by some over-interpretation errors due to the application of the Klinkenberg procedure for the determination of the intrinsic permeability starting from $k_{\text {app }}$. This allows stressing further the importance of properly distinguishing among crack and porous medum contributions (see Remark 2). After introducing this distinction, [12] evidenced that the crack flow was purely laminar and the slip flow contribution was quite negligible ${ }^{5}$ (i.e., no Klinkenberg effect occurred).

\footnotetext{
${ }^{5}$ This observation is in good agreement with litterature results concering slip flow conditions in micro-channels. Indeed, several authors [16] working on cooling systems for micro-electronic applications evidenced that the transition between no-slip and slip flow regimes should occur for a critical Knudsen equal to 0.001 (theoretical derivation). Turner [26] evidenced experimentally, however, that for parallel plates systems slip flow remains almost negligible up to Knudsen values of about 0.01 . If this second condition is retained, for air at atmospheric conditions slip should be neglected when the crack aperture becomes larger than $3.5 \mu \mathrm{m}$ (the mean free path of
}

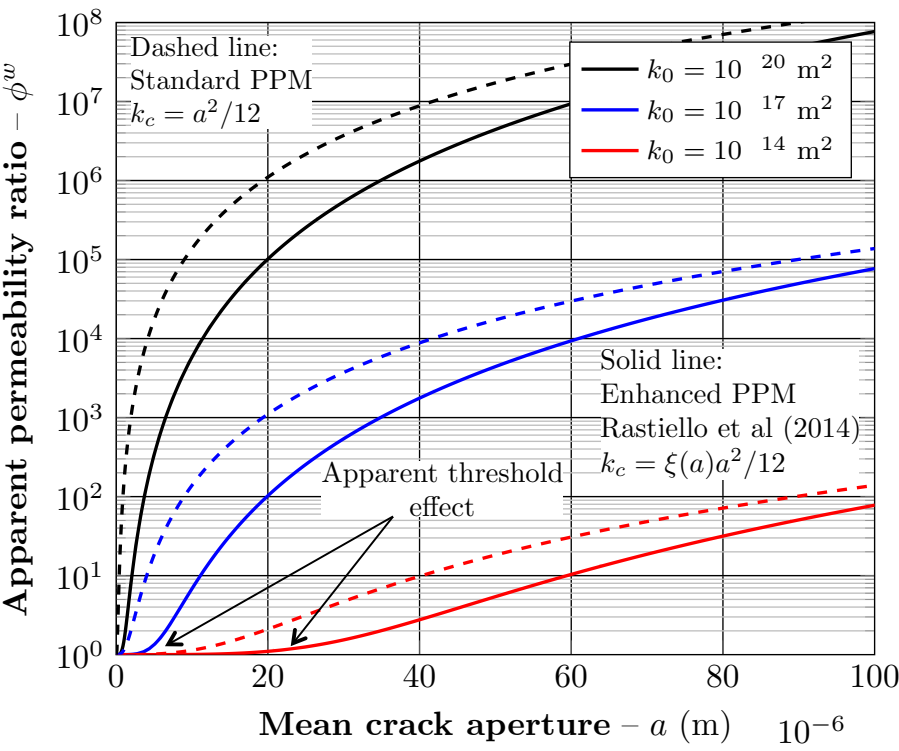

Figure 3: Evolution of the apparent permeability ratio $\phi^{w}=k_{\text {app }} / k_{0}$ with respect to the mean crack opening $(a)$ for different values of $k_{0}$. The crack permeability $k_{c}(a)$ is computed according to the standard parallel plates model (dashed line) or by the phenomenological relationship proposed in [21] (solid line). The crack is supposed $w=0.77$ $\mathrm{m}$ (as in experiments) in width, while the reference surface of the sample is $S_{0}=\pi w^{2} / 4$ (see Fig. 1).

\subsection{What crack opening displacement measure?}

The second keypoint arisen in this paper concerns a proper experimental estimation of crack opening displacements corresponding to flow measurements.

In the experiments by Wang et al. [28], the only geometrical information available were the mean apertures at mid-height of the sample, under loading $\left(a_{m}^{\text {loaded }}\right)$ and after unloading $\left(a_{m}^{\text {unloaded }}\right)$. This latter was measured just before performing the permeability test.

Figure 4 depicts the evolution of $\phi^{w}$ with respect to $a_{m}^{\text {unloaded }}$ and $a_{m}^{\text {loaded }}$, and provides a comparison with present results. The transmissivity ratio $\phi^{w}$ was estimated by using measured quantities only:

$$
\phi^{w}=\frac{Q}{Q_{0}}=\frac{T}{T_{0}}=1+\frac{Q_{c}}{Q_{0}}=1+\frac{T_{c}}{T_{0}}
$$

without requiring any modeling assumption concerning the crack geometry. Notice that, quantities $T$ and $T_{0}$ were both measured in [28], whereas the sole crack contribution $\left(T_{c}\right)$ was known in [21]. The transmissivity of the sound material was thus estimated considering $k_{0} \approx 10^{-18} \mathrm{~m}^{2}$ and $S_{0}$ as in [28].

Based on these results, one observes that:

molecules is of about $70 \mathrm{~nm}$ ). Now, provided the complex geometry of cracks in concrete one can imagine to increase this limit but, in any case, the Klinkenberg correction should be used carefully and in a small range of crack apertures. 
(i) When considering $a_{m}^{\text {loaded }}$ as the displacement variable, the ratio $\phi^{w}$ essentially evolves in two steps. It slightly increases for $a_{m}^{\text {loaded }} \quad 100 \mu \mathrm{m}$ approximately. When this crack opening limit is exceeded, the crack flow becomes more important and $\phi^{w}$ tends to non-linearly increase up to the end of the test. This transitional crack opening value is usually interpreted as a "threshold" corresponding to the full interconnection of the crack space.

(ii) This threshold condition is still present - but less clear - when considering $a_{m}^{\text {unloaded }}$ as the displacement variable. In that case, the first phase becomes more limited and $\phi^{w}$ increases in good agreement (values and overall tendency) with present results for $a_{m}$ larger than $50 \mu \mathrm{m}$. Although no statistical information can be obtained from results presented in [28] (they performed only one test), such comparison suggests that this second quantity should be preferred for their interpretation.

Once this choice is made, however, one still observes that for small opening values ( $a_{m}$ between $30 \mu \mathrm{m}$ and $\left.50 \mu \mathrm{m}\right)$ results are quite different ${ }^{6}$. This discrepancy can be partly attributed to some differences in the formulation of the tested concretes, of course, but parasite effects associated with the application of standard transfer permeability measurements cannot be excluded. In particular:

(i) Wang et al. [28] estimated the permeability after 10 days of flow monitoring. As a consequence, selfhealing phenomena [24] cannot be excluded.

(ii) Some reduction of the mass flow rate can also occur (for small $a_{m}^{\text {unloaded }}$ values) due to the presence of some contact areas within the crack (i.e., a non complete interconnection of the crack space) associated with the progressive mechanical re-closure of the crack after unloading the specimen.

These parasite effects can be strongly limited by adopting the real-time protocol presented in [21], as flow measurements are performed in real-time with loading and the time needed to achieve the stationary flow condition is very small. That means that a unique $a_{m}$ value can be associated to each flow measurement. Furthermore, this $a_{m}$ value does not evolve during flow measurement.

Remark 5. The standard procedure consisting in measuring a crack opening displacement before the permeability test might lead to inconsistencies as the crack geometrical configuration might change during the flow measurements. This observation suggests that the threshold effect should not be interpreted as an intrinsic crack property, but as an apparent effect mainly deriving from a misestimation of the crack opening level corresponding to the measured mass flow rate.

\footnotetext{
${ }^{6}$ In [21] the smallest explored value was of about $30 \mathrm{~m}$
}

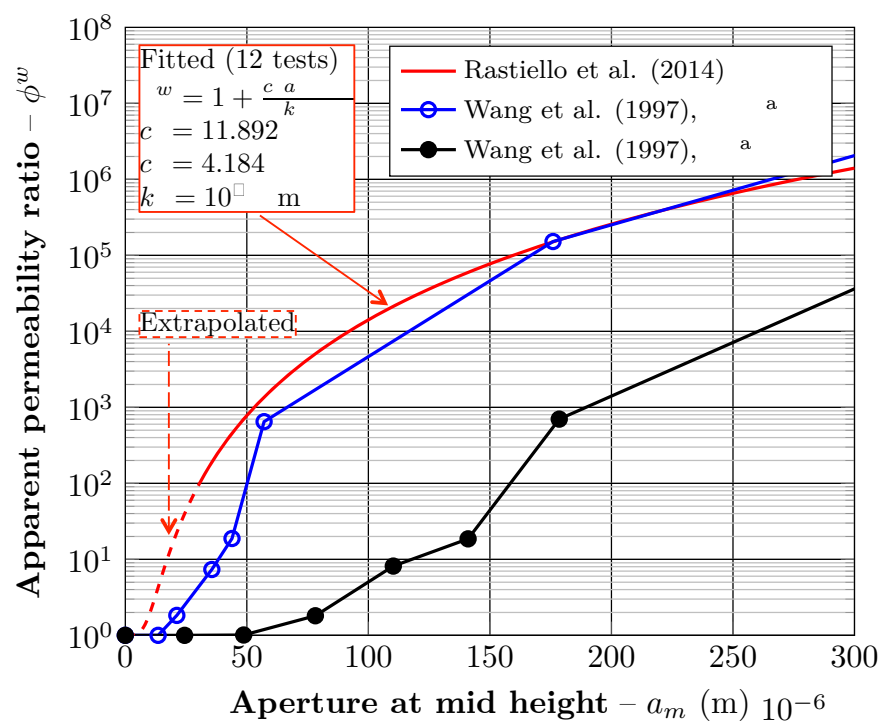

Figure 4: Comparison with results results provided by [28]. The solid red line corresponds to the evolution obtained considering for 0 the same value as in the cited reference work.

Remark 6. Till now we supposed that a single localized crack was present in the specimen. However, a further parasite effect associated with the use of standard experimental techniques to mechanically control the Brazilian tests can appear: the uncontrolled localization of secondary cracks. For instance, in [19] this occurred for $a_{m} \quad 34 \mu \mathrm{m}$. Under these conditions the opening process of the principal crack can be slowed down/stopped by the progressive opening and propagation of secondary cracks ${ }^{7}$. As a consequence, the crack opening displacement at mid-height of the crack is even less representative of the geometrical crack configuration.

Together with the previous remark concerning the use of the Klinkenberg method to air measures (see Remark 4), this second aspect can partly explain the strong differences observed when comparing present results with those obtained by Picandet et al. [19] for air flows through OC samples (Fig. 5).

It should be noticed that this comparison is possible because, under quasi-incompressible laminar conditions ${ }^{8}$ the ratio $\phi^{a}$ of the apparent air permeability of the cracked sample to the the initial permeability reads as in Eq. (6). Furthermore, this analysis is also consistent from a theoretical viewpoint, because theoretical considerations suggest that the crack permeability $k_{c}$ should be considered as an intrinsic crack properties (i.e., depending on the crack geometry only).

\footnotetext{
${ }^{7}$ Due to the use of a more effective control technique, in [21] secondary crack appears only for $\quad 300 \mathrm{~m}$. For that reason the tests were stopped once this displacement level was achieved.

${ }^{8}$ Under quasi-incompressible isothermal conditions, air flows through cracks can be still described according to a Darcy-like law, where the constant fluid density ( ) is replaced by the mean fluid density ( $)$ between the inlet and outlet sections of the crack/channel.
} 


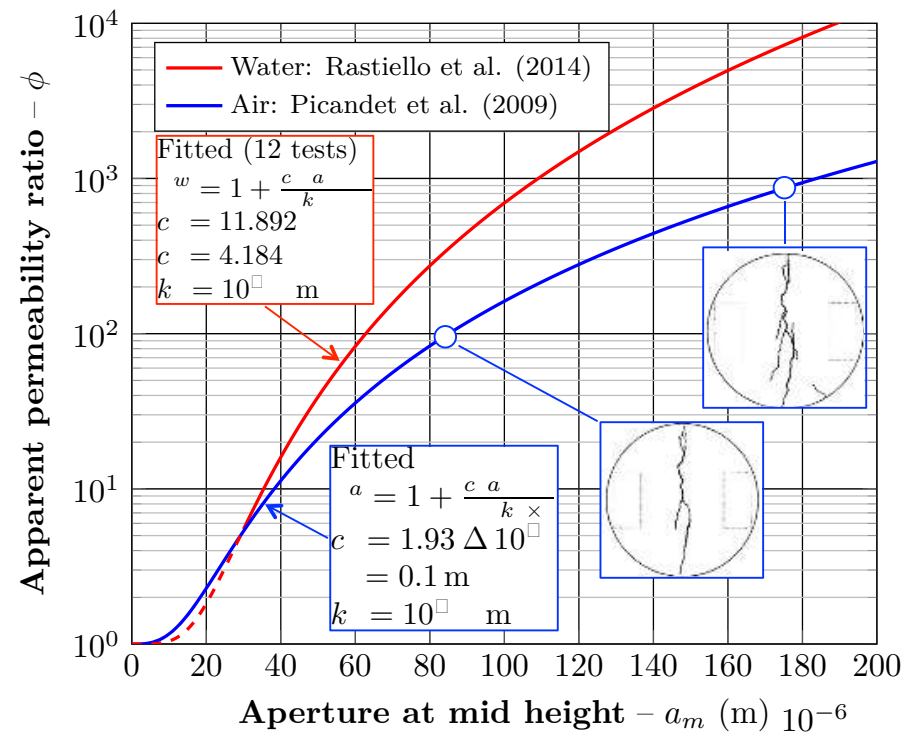

Figure 5: Comparison among results by [21] (water permeability) and [19] (air permeability) in terms of the apparent permeability ratio with respect to the crack opening at mid-height of the sample.

\subsection{What does such a threshold crack opening represents?}

As mentioned before, the existence of such a threshold crack opening effect is commonly explained as the result of an incomplete interconnection of the crack space. This can be justified in light of theoretical and experimental results obtained in the Rock Mechanics [18, 6, 30] context $^{9}$. Some critical conditions corresponding to no-flow are indeed possible, even though the crack opening in non null.

One should, however, distinguish between two main causes of non-interconnection:

(i) The first cause involves the presence of contact areas between the crack surfaces due to crack re-closure, after sample unloading and during permeability measurements.

(ii) The second cause concerns a still incomplete macrocrack localization.

\footnotetext{
${ }^{9}$ Based on the effective medium theory developed by Kirkpatrick [13] for the electrical conduction in random networks of resistors, Zimmerman and Bodvarsson [30] defined (for cracks is rocks) a percolation threshold in terms of the ratio of the crack space close due to contact areas ( ) to the total crack space ( ). In particular, they proposed a crack transmissivity - aperture law explicitly accounting for a no flow condition $(==0)$ for $=\geq 05$. Slightly different threshold values can be identified through other theories $[17,27]$. It should be noticed that, in a Continuum Damage Mechanics (CDM) context [14], the parameter could be also put into relation with a scalar damage variable $\left[\begin{array}{ll}0 & 1\end{array}\right]$. According to very simple one-dimensional CDM arguments, one can write:

$=\left(\begin{array}{ll}\quad & =1\end{array}=1 \quad\right.$. Hence, the threshold condition $\geq 05$ may be equivalently expressed in terms of the damage variable as: $\leq 05$.
}

The first case was discussed before. Concerning this second case, it is well known that concrete cracking is a complex phenomenon characterized by a progressive transition from diffuse damage to strain localization. Moderate apparent permeability increases before crack localization are, of course, possible due to micro-cracking [11]. However, they cannot be properly studied through experimental protocols based on displacement controlled Brazilian tests. This testing technique ensures that a single macro-crack rapidly propagates through the specimen once the load peak is reached. As a consequence of this, it is well suited for studying localized macro-cracks only and no information on the micro-cracking and strain localization phases can be obtained.

Remark 7. In both cases, it seems difficult to use these results in order to derive information about any threshold crack opening effect. Furthermore, the definition of such threshold crack opening is strictly dependent on what it is intended with the term "macro-crack". In this work, with this term, we defined an interconnected space where fluid flow is possible. If this interpretation is chosen, the evidence suggests that a threshold crack opening cannot be defined because the macro-crack still does not exists in this phase.

Remark 8. A final - and more general - remark concerns the difficulty of objectively defining a representative crack aperture for geometrically complex cracks (as in the case under consideration). In [21], we showed that instead of using the crack opening displacement $a_{m}$ one should prefer the mean crack opening $\bar{a}$, but such definition is still phenomenological. No information on the spatial distribution of local apertures inside the crack, on crack tortuousity and on the roughness the crack walls are available.

\section{Conclusive remarks}

This discussion paper introduced some considerations on the definition of a so-called threshold crack opening effect for the transfer properties of discretely cracked concrete. The existence of such a threshold effect is mentioned in most experimental works aiming to study the influence of discrete cracking on concrete permeability through Brazilian tests [28, 2, 19], and is classically explained as the result of an incomplete interconnection of the crack space for small crack opening levels.

The comparison among the experimental results obtained by [21] for water flow in cracking concrete and reference results of the literature [28] showed, however, that providing an objective identification of a crack opening displacement such that the crack flow is null, is not straightforward. It was shown that literature results could be affected by some technical and interpretation issues deriving from some limitations of classically adopted protocols, to the use of nonphysical quantities for representing the transfer properties 
of the cracked domain and to a misestimation of the crack opening level associated with flow measurements.

It was then stressed the importance of adequately distinguishing between crack and porous medium flow contributions for correctly interpret experimental results (when considering gas flows too). In [21], this was possible thanks to a more advanced experimental technique based on measuring transfer properties in real-time with the crack opening process (i.e., under loading). This allowed better controlling the crack opening process (the crack opening displacement was kept constant during flow measurements), thus improving the results quality and making their interpretation less ambiguous. The determination of the representative crack opening measure was, however, still based on phenomenological considerations as no information on the spatial distribution of local apertures inside the crack, on crack tortuousity and on crack walls roughness were available.

In order to further enhance these aspects, one could imagine developing more advanced experimental methods based on testing smaller specimens and ensuring more "controlled" crack geometric conditions. However, extracting small specimens from larger cracked sample without perturbation of the crack geometry is extremely difficult. Furthermore, the artificial reconstruction of micro-metric cracks is still a challenging topic. At the best of our knowledge, no protocols of this kind exist for concrete and concretelike materials. Further studies in this directions could be however useful.

In the meanwhile, in the Authors opinion it is important to make aware concerning too simplistic interpretations or possible over-interpretations of available experimental results.

\section{Acknowledgements}

The authors declare no potential conflicts of interest with respect to the research, authorship, and/or publication of this article. The authors received no financial support for the research, authorship, and/or publication of this article. Claude Boulay, former senior researcher at IFSTTAR and Université Paris-Est, is gratefully acknowledged.

\section{References}

[1] A. Akhavan, S.M.H. Shafaatian, and F. Rajabipour. Quantifying the effects of crack width, tortuosity, and roughness on water permeability of cracked mortars. Cement and Concrete Research, 42(2):313-320, 2012.

[2] C.M. Aldea, M. Ghandehari, S.P. Shah, and A. Karr. Estimation of water flow through cracked concrete under load. ACI Materials Journal, 97(5), 2000.

[3] Nathan Benkemoun, Hayder Al Khazraji, Philippe Poullain, Marta Choinska, and Abdelhafid Khelidj. 3-d mesoscale simulation of crack-permeability coupling in the brazilian splitting test. International Journal for Numerical and Analytical Methods in Geomechanics, pages n/a-n/a. nag.2749.

[4] J. Bodin, F. Delay, and G. De Marsily. Solute transport in a single fracture with negligible matrix permeability: 1 . fundamental mechanisms. Hydrogeology journal, 11(4):418-433, 2003.

[5] C. Boulay, S. Dal Pont, and P. Belin. Real-time evolution of electrical resistance in cracking concrete. Cement and Concrete Research, 39:825-831, 2009.

[6] Stephen R. Brown. Fluid flow through rock joints: The effect of surface roughness. Journal of Geophysical Research, 92(B2):1337, 1987.

[7] Ghislain De Marsily. Quantitative hydrogeology. Technical report, Paris School of Mines, Fontainebleau, 1986.

[8] Yining Ding, Dong Li, and Yulin Zhang. Quantitative analysis of macro steel fiber influence on crack geometry and water permeability of concrete. Composite Structures, 187:325 - 335, 2018.

[9] A. Djerbi, S. Bonnet, A. Khelidj, and V. Baroghel-bouny. Influence of traversing crack on chloride diffusion into concrete. Cement and Concrete Research, 38(6):877 - 883, 2008.

[10] Mohamad Ezzedine El Dandachy, Matthieu Briffaut, Frédéric Dufour, and Stefano Dal Pont. Coupling by means of strong discontinuity approach between crack opening and gas permeability for concrete. S25 Réponses des matériaux et des structures de Génie Civil aux sollicitations sévères, 2015.

[11] Meghdad Hoseini, Vivek Bindiganavile, and Nemkumar Banthia. The effect of mechanical stress on permeability of concrete: A review. Cement and Concrete Composites, 31(4):213 $-220,2009$.

[12] Xavier Jourdain. Etude numérique méso-macro des propriétés de transfert des bétons fissurés. $\mathrm{PhD}$ thesis, LMT-Cachan, École Normale Supérieure de Cachan, 2014.

[13] S. Kirkpatrick. Percolation and conduction. Reviews of Modern Physics, 45(4):574, 1973.

[14] Jean Lemaitre and Rodrigue Desmorat. Engineering damage mechanics: ductile, creep, fatigue and brittle failures. Springer Science \& Business Media, 2005.

[15] M. Matallah and C. La Borderie. 3d numerical modeling of the crack-permeability interaction in fractured concrete. 2015.

[16] G.L. Morini, M. Spiga, and P. Tartarini. The rarefaction effect on the friction factor of gas flow in microchannels. Superlattices and Microstructures, 35(3-6):587 - 599, 2004. Eurotherm 75 'Microscale Heat Transfer 2'.

[17] A.N.M. Obdam and E.J.M. Veling. Elliptical inhomogeneities in groundwater flow - an analytical description. Journal of Hydrology, 95(1):87 - 96, 1987.

[18] Nadir Patir and H. S. Cheng. An Average Flow Model for Determining Effects of Three-Dimensional Roughness on Partial Hydrodynamic Lubrication. Journal of Lubrication Technology, 100(1):12, jan 1978.

[19] V. Picandet, A. Khelidj, and H. Bellegou. Crack effects on gas and water permeability of concretes. Cement and Concrete Research, 39(6):537 - 547, 2009.

[20] S. Rahal, A. Sellier, and G. Casaux-Ginestet. Finite element modelling of permeability in brittle materials cracked in tension. International Journal of Solids and Structures, 113-114:85 - 99, 2017.

[21] G. Rastiello, C. Boulay, S. Dal Pont, J.L. Tailhan, and P. Rossi. Real-time water permeability evolution of a localized crack in concrete under loading. Cement and Concrete Research, 56(0):20 - 28, 2014.

[22] G. Rastiello, C. Desmettre, J.-L. Tailhan, P. Rossi, J.-P. Charron, and S. Dal Pont. Modeling of fluid leakage through multicracked RC structural elements using a numerical probabilistic cracking approach. Materials and Structures, 2015.

[23] G. Rastiello, J.-L. Tailhan, P. Rossi, and S. Dal Pont. Macroscopic probabilistic cracking approach for the numerical modelling of fluid leakage in concrete. Annals of Solid and Structural Mechanics, pages 1-16, 2015.

[24] Hans-Wolf Reinhardt and Martin Jooss. Permeability and selfhealing of cracked concrete as a function of temperature and 
crack width. Cement and Concrete Research, 33(7):981-985, 2003.

[25] D.T. Snow. A Parallel Plate Model of Permeable Fractured Media. PhD thesis, University of California at Berkley, 1969.

[26] Stephen E. Turner, Lok C. Lam, Mohammad Faghri, and Otto J. Gregory. Experimental investigation of gas flow in microchannels. J. Heat Transfer, 126(5):753-763, 2004.

[27] J.B. Walsh. Effect of pore pressure and confining pressure on fracture permeability. International Journal of Rock Mechanics and Mining Sciences 83 Geomechanics Abstracts, 18(5):429 435, 1981.

[28] K. Wang, D.C. Jansen, S.P. Shah, and A.F. Karr. Permeability study of cracked concrete. Cement and Concrete Research, 27(3):381-393, 1997.

[29] P.A. Witherspoon, Y.S.Y Wang, K. Iwai, and J.E. Gale. Validity of cubic law for fluid flow in a deformable rock fracture. Water Research Resources, 16(6):1016-1024, 1980.

[30] R.W. Zimmerman and G.S. Bodvarsson. Hydraulic conductivity of rock fractures. Transport in Porous Media, 23:1-30, 1996. 